\title{
Las TIC en la investigación de las Licenciaturas en Educación Infantil en Colombia
}

\section{ICT in the Research of the Bachelor's Degrees in Child Education in Colombia}

\author{
Marlon Marcos Molina Manosalva \\ Universidad del Atlántico \\ mmmolina@mail.uniatlantico.edu.co \\ Colombia
}

\author{
María Esther Tobos Valderrama \\ Universidad del Atlántico \\ mariatobos@mail.uniatlantico.edu.co \\ Colombia
}

\section{Resumen:}

El presente estudio tiene como objetivo develar el estado en que se encuentra la producción investigativa con relación a las Tecnologías de la Información y la Comunicación (TIC), en las Licenciaturas en Educación Infantil en las universidades de la región central de Colombia en el período 2009-2019, para lo cual se propone en primer lugar, evidenciar la concepción de TIC en los trabajos de grado analizados, para posteriormente identificar los temas alrededor de los que han girado dichas investigaciones. El estudio se desarrolla desde el paradigma cualitativo inspirado en la hermenéutica contemporánea, pues se busca comprender el significado en los trabajos de grado, en su contexto y devenir histórico, tomando como proceso metodológico el Círculo Hermenéutico, en tanto que es un recurso explicativo de tipo dialógico. Se emplean como instrumentos de recolección de información: la Matriz Bibliográfica, la Matriz por Temas-Problemas y la Ficha Resumen o Ficha Analítica. Como resultado se encuentra que la concepción de TIC en los trabajos de grado analizados es instrumental. Así mismo, se evidencian cuatro tendencias alrededor de las cuales han girado las investigaciones en TIC: 1) las TIC como un medio para enseñar en la infancia, 2) las TIC en las interacciones de la infancia, 3) políticas de las TIC en la educación infantil y 4) conocimiento de las TIC en educación infantil.

Palabras clave: Estado del arte; Tecnologías de la Información y Comunicación (TIC); Educación Infantil.

\begin{abstract}
:
The objective of this study is to reveal the state of the research production in relation to Information and Communication Technologies (ICT), in the Bachelor Degrees in Early Childhood Education in the Universities of the Central Region of Colombia in the period 2009 -2019. To achieve this we propose, in the first place, to show the conception of ICT in the degree projects analyzed, to later identify the issues around which said investigations have revolved. The study is developed from the qualitative paradigm inspired by contemporary hermeneutics, since it seeks to understand the meaning in degree projects, in its context and historical development, taking the Hermeneutical Circle as a methodological process, as it is an explanatory resource of a dialogic type. The information gathering instruments were: the Bibliographic Matrix, the Matrix by Issues-Problems, and the Summary Card or Analytical File. As a result, we found that the conception of ICT in the Degree Projects analyzed is instrumental. Likewise, there are four trends around which ICT research has revolved: 1) ICT as a teaching means in childhood, 2) ICT in childhood interactions; 3) ICT policies in early childhood education, and 4) knowledge of ICT in early childhood education.
\end{abstract}

Keywords: State of the Art; Information and Communication Technologies (ICT); child education.

Recibido: 30/10/2020 | Aceptado: 07/12/2020 | Publicado: 08/01/2021

| pág. 51 - 69 


\section{Las TIC en la investigación de las Licenciaturas en Educación Infantil en Colombia}

\section{| Introducción y propósito del estudio}

La presente investigación da cuenta de una parte de los resultados obtenidos dentro del estudio titulado "Estado de la Investigación en la Formación Docente para la Educación Infantil en Universidades de la Región Caribe y Centro del País”, de igual forma refleja el análisis a los corpus del texto expresados en los Trabajos de Grado, en adelante TG, relacionados con las TIC en cinco universidades de la región central de Colombia, en la década comprendida entre 2009 y 2019, para lo cual se valora el aporte investigativo expresado por quienes en su momento se formaban como docentes en el campo de la Educación Infantil. Además, se genera una serie de reflexiones que dan cuenta de la concepción de TIC en la Educación Infantil, así como de algunas tendencias alrededor de las cuales han girado las investigaciones en TIC. Es importante señalar que el presente trabajo de investigación se inscribe en un paradigma interpretativo; por tanto, es un compromiso ético ineludible hacer explícita la perspectiva teórica desde la cual se valoran los TG que incursionan en este campo temático.

El acercamiento a la conceptualización de Tecnologías de la Información y la Comunicación, establece un precedente para el análisis, con definiciones como la propuesta por García (como se citó en Longoria, 2008) que llama TIC "al conjunto de tecnologías que permiten la adquisición, producción, almacenamiento, tratamiento, comunicación, registro y presentación de informaciones, en forma de voz, imágenes y datos contenidos en señales de naturaleza acústica, óptica o electromagnética” (p. 12), mientras que Cabero (como se citó en Najar, 2016), sostiene que las TIC: 
Son las que giran en torno a tres medios básicos: la informática, la microelectrónica y las telecomunicaciones; pero giran, no sólo de forma aislada, sino lo que es más significativo, de manera interactiva e interconexionadas, lo que permite conseguir nuevas realidades comunicativas ( $p$. 10).

En este sentido, se entienden como TIC, al conjunto de todas aquellas herramientas, recursos o dispositivos que permiten el procesamiento, la gestión y el acceso a la información, representada en diversos formatos ya sea texto, imagen o sonido, valiéndose de equipos como computadores, televisores, radios, teléfono, tabletas, etc.

En la actualidad no hay duda de que las TIC han entrado a jugar un papel fundamental en la vida de las personas, al influenciar la cotidianidad y la manera en que éstas se comunican y se relacionan. No en vano indica Lara (2017) que "las TIC coexisten en la familia, la escuela, bibliotecas y portadas en el cuerpo de los sujetos como una extensión” (p. 7). Desde que las TIC surgieron a finales del siglo pasado, han venido experimentado un vertiginoso crecimiento, lo que a su vez ha ocasionado un gran interés en conocer la manera en que éstas pueden ser empleadas en los diferentes campos de la existencia humana, desde la educación, el trabajo y la sociedad en general, a fin de mejorar la calidad de vida de las personas.

En el caso de Colombia, el gobierno ha asumido las TIC en el ámbito educativo, como una parte fundamental para el desarrollo económico y social del país, a fin de aumentar la competitividad y productividad de éste, estableciendo toda una agenda política a través del Ministerio de las TIC, que mediante la ley 1341 de 2009, determinó toda la normatividad correspondiente al uso e implementación de estas tecnologías en el territorio colombiano. Así mismo, se han creado diferentes estrategias como Agenda de conectividad que permitió masificar el uso de las TIC en la población, y las alianzas entre el Ministerio de las TIC y el Ministerio de Educación Nacional 
(MEN) a través de programas como Computadores para Educar, con el que se buscó brindar acceso a la tecnología en la comunidades educativas a fin de disminuir la brecha digital en el país.

Aunque las estrategias descritas anteriormente se constituyen en una inversión de gran valor, hay que resaltar que éstas solo permiten garantizar el acceso de la población a unos recursos físicos pero no a un conocimiento en el uso de los mismos, lo que hace necesaria una alfabetización tecnológica en la población colombiana, es así como el MEN con la creación de los estándares básicos de tecnología e informática busca superar en parte tales dificultades, haciendo que tanto estudiantes como docentes se formen en el uso de las diversas tecnologías. De igual forma mediante la Resolución 02041 de 2016, el MEN estableció el desarrollo de competencias en el uso de las Tecnologías de la Información y Comunicación en los maestros en formación, como uno de los requisitos que deben cumplir las universidades para obtener el Registro Calificado de sus Programas de Licenciatura.
En el campo de la educación como bien dicen Abadía, Quiceno y Rivera (2013), las TIC “pueden transformar y mejorar las prácticas educativas, al presentarse como una alternativa de apoyo y complementariedad a las prácticas presenciales, ofreciendo nuevas posibilidades y recursos para enriquecer el proceso de enseñanza y aprendizaje" (p. 14). $Y$ es que, con la implementación de las TIC en campo educativo, se busca la creación y exploración de nuevos y mejores ambientes y metodologías de aprendizaje.

Por su parte, el desarrollo de las TIC en la Educación Infantil ha permitido el surgimiento de procesos de enseñanza aprendizaje innovadores, en los que el uso de tecnologías en experiencias de gamificación han posibilitado el desarrollo de conocimientos y habilidades en los más pequeños de una manera mucho más acogedora, y es que, como sostienen Ching-Ting, Ming-Chaun y Chin-Chung (citados en Escorcia y Gómez, 2019), no se puede olvidar que "los niños de hoy en día son parte de la generación de nativos digitales pues, viven en un mundo que está rodeado por 
tecnologías y al igual hacen uso de estas en su vida” (p. 12), aunque hay que tener presente que tal afirmación no se puede generalizar ya que cada infancia tiene unas condiciones particulares por lo que el acceso a los recursos tecnológicos estará condicionado por múltiples factores.

Sin embargo, aún con estos planteamientos, Rueda (como se citó en Parra, 2012) manifiesta que el “impacto de la investigación producida en las Universidades y los programas masivos de educación en el campo de las TIC es muy incipiente" (p. 156), a pesar de la importancia que las TIC tienen para la sociedad y para la educación de los más pequeños en particular, lo que lleva a pensar en la necesidad de conocer ¿qué se ha investigado con relación a las TIC en los Programas de Formación para la Educación Infantil en las universidades de la región central de Colombia en la última década?

\section{|Metodología}

Indagar sobre las investigaciones realizadas en torno a las TIC en las Licenciatura en Educación Infantil, implica incursionar en la realidad social de la formación docente para quienes se desempeñarían en este ámbito de la educación, fenómeno que se expresa en los informes de los TG. Este hecho la enmarca en la investigación cualitativa inspirada en la hermenéutica contemporánea de Gadamer (1993), enriquecido por Ricoeur (1991), que busca comprender los significados en los textos TG en su contexto y devenir histórico. Se emplea como metodología el Circulo Hermenéutico que, al ser un recurso explicativo de tipo dialógico, permite la interrelación entre las expectativas y prejuicios del investigador con la realidad social objeto de estudio, que como dice Tobos (2016) citando a Schleiermacher (2007), permite indicar un modo de proceder, una postura de pensamiento, que designa la estructura circular de la intelección o entendimiento como un proceso circular a manera de espiral (virtuoso) de entendimiento del objeto de investigación donde se definen tres grandes fases 0 etapas: a) la pre-comprensión, b) la interpretación y la comprensión, y c) la construcción de sentido o construcción teórica. 
Para la población y foco de identificando una producción total la investigación, de manera intencional se seleccionó un grupo de 1014 informes de TG relacionados con la Educación Infantil.

de universidades que cumplieran con tres premisas: primero, que representaran la región del centro del país, segundo, que ofertaran los programas de Educación Infantil, y tercero, que dichas universidades dispusieran de los llamados repositorios institucionales que permiten el acceso a la producción investigativa en ellas desarrollada. De tal manera que finalmente se seleccionaron cinco universidades: la Pontificia Universidad Javeriana, la Universidad de Antioquia, la Universidad Pedagógica Nacional, la Universidad de la Sabana y la Universidad Tecnológica de Pereira. Asimismo se tomó como lapso de referencia el periodo 2009-2019,

De los 1014 informes de TG, se encontró que solo 43 de ellos hacían referencia a las TIC, los cuales fueron tomados como una muestra inicial (Tabla 1). Posteriormente, empleando el concepto expuesto por Glasser y Strauss (1967) en la teoría fundada de muestra teórica por saturación, que consiste en un proceso simultáneo de comparación constante y muestreo teórico como guía que ilumina el trabajo investigativo de recolección y análisis de los datos que emergerían en sucesivos estadios en el proceso investigativo, la muestra se reduce finalmente a 13 informes de TG (Tabla 2).

Tabla 1.

Distribución de la muestra inicial por universidad

\begin{tabular}{ccc}
\hline Universidad & No (TG) & Repositorio Institucional \\
\hline Pontificia Universidad Javeriana & 4 & https://repository.javeriana.edu.co/ \\
Universidad de Antioquia & 4 & http://bibliotecadigital.udea.edu.co/ \\
Universidad Pedagógica Nacional & 4 & http://repositorio.pedagogica.edu.co/ \\
Universidad de la Sabana & 7 & https://intellectum.unisabana.edu.co/ \\
Universidad Tecnológica de Pereira & 24 & http://repositorio.utp.edu.co/dspace/ \\
\hline Total de TG & 43 & \\
\hline
\end{tabular}


Las TIC en la investigación de las Licenciaturas en Educación Infantil en Colombia

Molina Manosalva y Tobos Valderrama

Tabla 2.

Distribución de la muestra final por universidad

\begin{tabular}{cc}
\hline Universidad & No (TG) \\
\hline Pontificia Universidad Javeriana & 4 \\
Universidad de Antioquia & 2 \\
Universidad Pedagógica Nacional & 3 \\
Universidad de la Sabana & 2 \\
Universidad Tecnológica de Pereira & 2 \\
\hline Total de TG & 13 \\
\hline
\end{tabular}

Con relación a los instrumentos empleados para la recolección de la información se tuvo en cuenta en primer lugar, la Matriz Bibliográfica con la cual se buscó extraer los datos bibliográficos de los TG analizados a fin de sistematizarlos y organizarlos; en segundo lugar, la Matriz por Temas-Problemas que agrupaba los títulos de los TG por características comunes, a fin de identificar los temas alrededor de los cuales han girado las investigaciones con relación a las TIC en los programas de Educación Infantil en las universidades del centro del país, y en tercero, la Ficha Resumen con la que se buscó comprender el todo y cada una de las partes de los TG, a fin de develar la concepción de TIC que se encontraba en ellos.

\section{Análisis de los datos y resultados de la investigación}

En un primer momento y tomando como referencia los 43 informes de TG, se hizo un análisis de los temas problemas objeto de la investigación empleando la Matriz por TemasProblemas de las que emergen cuatro tendencias alrededor de las cuales han girado las investigaciones en los TG con relación a las TIC en las Licenciaturas en Educación Infantil en las universidades del centro del país durante la última década. Entre estas tendencias están: 1) las TIC como medio para enseñar en la infancia, 2) las TIC en las interacciones de la infancia, 3) políticas de las TIC en la educación infantil y 4) conocimiento de las TIC en educación infantil. 
Con el procedimiento de muestreo teórico y comparación constante, el estudio se optimizó en un total de 13 TG, cuyos temas, como dicen Strauss y Corbin (2002), se encontraban repetidamente presentes (o en algunas situaciones, notablemente ausentes) y actuaban como condiciones que le daban variación a las cuatro tendencias, a cada una de las cuales se le hizo la Ficha Resumen. Considerando lo anterior se obtuvo el siguiente análisis:

\section{Las TIC como un medio para enseñar en la infancia}

Esta es la tendencia que más se ha abordado en los TG analizados; son 34 investigaciones que se enfocan en caracterizar las TIC como un medio, ya sea para enseñar a los estudiantes los contenidos de una disciplina como Educación Física, Español, Educación Artística, Inglés, Geometría, entre otras o, para desarrollar habilidades en el lenguaje, la escritura, comprensión lectora, etc.

La universidad que más desarrolla esta tendencia es la Universidad Tecnológica de Pereira (UTP), con 24 TG. En este sentido, se podría decir que es la única tendencia con relación a las TIC que se desarrolla en dicha universidad, situación que puede estar relacionada con la amplia trayectoria que en el campo de la tecnología tiene este centro de formación que, en el año 2016 firmó el convenio 1046 con el Ministerio de Tecnologías de la Información y Comunicaciones, quien la consideró un Centro de Pensamiento en este campo del conocimiento.

\section{Las TIC en las interacciones de la infancia}

Esta tendencia de investigación ha sido poco desarrollada; solo fue posible identificar cuatro TG que abordan las TIC en la interacción de la Infancia, dos de ellos realizados en la Universidad de Antioquia, otro en la Universidad de la Sabana y un último en la Pontificia Universidad Javeriana. Parece ser, tal como lo dicen Correa y Cuello (2016), que "la mayoría de pesquisas realizadas hasta ahora muestran una fuerte tendencia a indagar estos asuntos con la población adolescente siendo muy pocos los estudios que han centrado exclusivamente la atención en los niños” (p. 6). 
Evidentemente, este es un tema que apenas se encuentra en desarrollo, aun cuando no se puede negar la gran influencia que actualmente ejercen las TIC en la vida de los más pequeños, pues forman parte de su cotidianidad. Como afirma Lara (2017), las interacciones mediadas por TIC "por un lado propician el aprendizaje autónomo, posibilitan que el sujeto pueda definir fines de aprendizaje partiendo de sus intereses, posibilita nuevas formas de socialización” (p. 61), lo que llega incluso a cambiar la forma en que los pequeños se relacionan con sus pares y adultos. En este mismo sentido, Valencia (2015) plantea "que entre más simétrico sea el grupo a nivel de intereses y edad será más alto el nivel de interacción y conexión entre dos o más integrantes" (p. 88), lo que permite a su vez configurar nuevos modos y formas de ser infantes, superando así los límites de espacio y tiempo antes impuestos. Es evidente que son el juego y el entretenimiento los que determinan la relación TIC e infancia, ya que en ellos es donde los niños encuentran utilidad a las TIC.

\section{Políticas de TIC en la educación infantil}

Esta tendencia de investigación en las TIC fue la menos abordada, solo se identificaron dos TG, desarrollados en la Pontificia Universidad Javeriana. Ambas investigaciones se refieren a las políticas sobre TIC en Educación y coinciden en señalar que "el papel del gobierno en las políticas públicas ... se ha centrado en infraestructura y cierre de brechas digitales" (Avilez, 2018, p. 60), evidenciando de alguna manera que las políticas del Gobierno Nacional en relación a las TIC en la educación infantil han estado enfocadas sobre todo hacia una mera dotación de los centros educativos con diversos recursos tecnológicos tales como computadores, tabletas, Internet, etc. Al parecer no se ha entendido que no se trata solo de equipar las aulas de clases, como dice Avilez (2018), "sino que se trata de brindar una apropiación en calidad de materiales y estrategias para que las prácticas pedagógicas (puestas en juego) estén mejor mediadas por las tecnologías digitales” (p. 71).

Tal como lo dice Gil y Maldonado (2009) en Colombia "aún no existe 
una política pública que contemple la ciencia y la tecnología dentro de la formación de la primera infancia” (p. 89), lo que hace difícil para las escuelas vincular estas prácticas a su cotidianidad ya que los docentes en su mayoría no tienen la formación pertinente en este campo del saber, aun cuando hoy es imprescindible sumergir a los niños en estos conocimientos, a fin que desarrollen las habilidades que el mundo actual exige en materia de ciencia y tecnología.

\section{Conocimiento de las TIC en educación infantil}

Esta tendencia de investigación en las TIC ha sido muy poco desarrollada, solo fue posible evidenciar tres TG relacionados con ella, dos realizados en la Universidad Pedagógica Nacional y otro en la Universidad de la Sabana. En dichas investigaciones se pudo identificar dos aspectos con relación al conocimiento de las TIC en educación infantil, uno desde los docentes en formación de semestres iniciales y finales, y otro desde una propuesta para los niños que transitan por el preescolar.
En cuanto al primer aspecto, Camacho y Contreras (2016) refiriéndose a los docentes manifiestan que "la gran mayoría tuvieron un acercamiento a las TIC durante su proceso escolar ... entre los 8 y 12 años aproximadamente" (p. 100). Asimismo, fue posible evidenciar grandes diferencias entre los docentes que inician la formación y quienes la finalizan; los docentes de semestres iniciales, refiriéndose a las TIC dicen que, "no consideran que el uso de estas herramientas sea positivo, así como bueno para los niños " (Yepes, 2014, p. 49), de igual forma "no consideran necesaria la tecnología para trabajar en el aula, argumentan que por muchos años no se ha usado y ha funcionado el aprendizaje” (Yepes, 2014, p. 50), situación que se puede deber en parte a la poca, nula o desarticulada formación recibida en el uso pedagógico de las TIC en los primeros semestres.

Mientras que los docentes de semestres finales, como lo indican Camacho y Contreras (2016), sí reconocen "la necesidad de un conocimiento en TIC como importante y necesario” (p. 137), 
lo que hace evidente entonces que al tener ya un conocimiento sobre las TIC bien sea por la formación recibida o por otro medio, los docentes de semestres avanzados sí conciben la relevancia de las TIC en la educación y en su vida laboral, aunque la mayoría de ellos manifiesta tener dificultades para incluirlas en su hacer pedagógico.

En el segundo aspecto, con relación a la implementación de una propuesta para promover la educación en TIC, Reyes (2016) manifiesta que con dicha propuesta se crea "la posibilidad de integrar todos los aspectos de la vida a la Educación en Tecnología, permitiendo que el niño(a) haga uso de sus conocimientos tanto dentro como fuera del aula” (p. 126). Asimismo, se logran desarrollar las habilidades cognitivas, de lenguaje y afectividad, entre otras, en los más pequeños, pues, aunque los niños tienen contacto frecuente con la tecnología desde muy temprana edad, no todos tienen las mismas oportunidades de acceso a todo tipo de dispositivos, y no se debe olvidar que el recurso tecnológico por sí solo no es garantía de éxito, por lo que es necesaria una formación en tales aspectos.

\section{Concepción de las TIC en los TG de Educación Infantil}

Con respecto a la concepción de las TIC en los TG de Educación Infantil, en las Fichas Resumen de las investigaciones analizadas predomina una concepción instrumental, que se evidencia en la forma como se refieren a éstas en el desarrollo de los textos; dicha afirmación en algunos momentos hace referencia a las TIC como herramientas. Así lo confirman Abadía, Quiceno y Rivera (2013) cuando, refriéndose a las TIC, manifiestan que "se puede decir que ellos ven esta herramienta como una posibilidad” (p. 82). De igual forma, Yepes (2014) dice que "existen otros materiales y herramientas que pueden ser utilizadas en el aula y no son tecnológicas” (p. 50). Asimismo, Ardila (2014) afirma que "la tecnología, se considera una herramienta útil que puede ser usada por los maestros” (p. 106). De la misma manera Lamilla, Rodríguez y Salgado (2015), 
refiriéndose a las TIC, dicen que son "entendidas de ahora en adelante como herramientas tecnológicas" (p. 10).

En otros contextos las TIC son asumidas como artefactos tecnológicos, ejemplo de ello es la afirmación de Ardila (2014) cuando plantea que "en algunas entrevistas y charlas con docentes, la postura del uso de tecnología para desarrollar las habilidades en el arte, se inclinan más hacia evitar el trabajo con los diferentes aparatos electrónicos” (p. 106). De igual forma Bustos, Gómez y Galeano (2019), igualando las TIC con los aparatos tecnológicos, dicen: "esta propuesta se realizará ... utilizando las TIC que brinda la institución como es la sala de sistemas con computadores de escritorio o computadores portátiles en el aula de clases, video beam, tablets, conexión a Internet, torre de sonido, y T.V.” (p. 71).

En otros momentos las TIC son consideradas un recurso tecnológico. Así lo afirma Lara (2017), al decir que "las TIC operan como recurso de difusión global” (p. 60 ); en este mismo sentido, Ardila
(2014) también plantea el desarrollo de la clase de educación artística "utilizando como elemento de mediación recursos tecnológicos", y de igual forma Bustos, Gómez y Galeano (2019) consideran que "los recursos tecnológicos con los que cuenta la institución, como Internet, computadores, tabletas ... son favorables y motivantes hacia el aprendizaje del inglés” (p. 22).

Finalmente, las TIC en otros espacios son consideradas un medio para lograr un fin. Así lo evidencian Abadía, Quiceno y Rivera (2013) cuando presentan los resultados de su investigación y dicen "en cuanto a los estudiantes mostraron un alto nivel de motivación en la implementación de la secuencia didáctica mediada por TIC” (p. 91).

Todo lo anterior lleva a pensar que esta concepción instrumental de las TIC de los que en su momento transitaban en la formación como Educadores de la Infancia y de quienes ejercían como docentes en centros educativos, coincide con los planteamientos que sobre las mismas hace el Ministerio de 
las Tecnologías de la Información y las Comunicaciones, cuando al referirse a las TIC dice "son el conjunto de recursos, herramientas, equipos, programas informáticos, aplicaciones, redes y medios; que permiten la compilación, procesamiento, almacenamiento, transmisión de información como: voz, datos, texto, video e imágenes” (Art. 6 Ley 1341 de 2009). Es precisamente allí, donde puede estar la causa de que en los docentes aún persista esta concepción instrumental de las TIC, pues desde que éstas entraron en auge en el siglo pasado, los Gobiernos a través del Ministerio de las TIC y el Ministerio de Educación Nacional, han diseñado y puesto en marcha políticas y programas como Agenda de Conectividad, Computadores para Educar, entre otros, a fin de brindar un mayor acceso y uso de las tecnologías, y así cerrar la brecha digital que existe en la población colombiana. Pero estas medidas han sido insuficientes, pues solo se han enfocado en equipar las escuelas con herramientas de cómputo y acceso a Internet, sin comprender que estos recursos por sí solos no garantizan el aprendizaje.
Por otra parte, como lo sostiene Camacho y Contreras (2016), "Tampoco es suficiente con expedir normativas que planteen la inclusión de las TIC y que no sobrepasen el papel” (p. 139), ya que en ocasiones las normativas emitidas por el Ministerio de Educación Nacional han buscado establecer todas unas exigencias a cumplir por parte de los Centros de Formación Docente, sin hacer las dotaciones y el acompañamiento necesario para que en éstos se brinden a los futuros maestros la formación tanto instrumental como pedagógica pertinente en TIC que estos requieren, ya que, como lo afirman Lamilla, Rodríguez y Salgado (2015), para lograr incorporar las TIC de manera adecuada se requiere un equipamiento de herramientas básicas necesarias para el uso de las mismas y capacitación a los docentes en el ámbito tecnológico y pedagógico (p. 11).

El carácter instrumental de las TIC se debe en parte al desconocimiento y al bajo dominio pedagógico que los docentes tienen de éstas, por lo que resulta necesario que hoy las TIC sean comprendidas y asumidas 
con el carácter pedagógico que les compete, tanto en los currículos de las escuelas como en el de las universidades, pues solo en la medida en que cambie la concepción de las TIC en los maestros se podrá superar dicha instrumentalización en la que han caído y de esta manera cumplir la finalidad para la cual fueron vinculadas al campo educación, que es precisamente la mejora en la calidad.

\section{|Conclusiones}

Con relación a la producción investigativa desarrollada en las Licenciaturas en Educación Infantil en las universidades del centro de Colombia entre el 2009 y el 2019, es posible concluir que los temas investigados alrededor de las TIC en los TG giran alrededor de cuatro tendencias, a saber. Primera, las TIC como un medio para enseñar en la infancia, que fue la tendencia más desarrollada en las investigaciones con 33 TG; la Universidad Tecnológica de Pereira fue la que más desarrolló esta tendencia con 24 TG. Segunda, las TIC en las interacciones de la infancia, que es una tendencia en pleno desarrollo, lo que se evidencia en la poca producción investigativa con la que se cuenta, debido a que las realizadas han tomado en su mayoría niños de educación básica primaria $y$ secundaria, dejando fuera el preescolar. Tercera, políticas de las TIC en la educación infantil, que al igual que la anterior tendencia apenas se encuentra en desarrollo, pues tal como lo plantean Gil y Maldonado (2009) "aún no existe una política pública que contemple la ciencia y la tecnología dentro de la formación de la primera infancia” (p. 89). Finalmente, la cuarta es el conocimiento de las TIC en Educación Infantil, aspecto en el que se hace necesaria una amplia formación de los docentes pues, aunque manifiestan tener conocimiento sobre este campo, no saben cómo incluirlo en su quehacer diario.

En este sentido, reconocer y profundizar en las tendencias de investigación con relación a las TIC se hace completamente pertinente para el momento actual, en el que la lógica de la escuela pareciere no estar respondiendo a las exigencias y 
necesidades del contexto en el que se encuentran inmersas. Esta situación invita a replantear otras formas de asumir los procesos de enseñanza aprendizaje en las aulas, pues aun cuando hay un notable número de investigaciones con relación las TIC como medio para la enseñanza de conocimientos disciplinares 0 habilidades, es necesario que se evalúen los resultados alcanzados con estas investigaciones a fin de determinar la pertinencia $y$ los aportes que han dejado. De igual forma, es necesario abordar las otras tendencias que apenas se encuentran en desarrollo $y$ que se convierten en una invitación y un reto para quienes se forman como educadores de la infancia y de la educación en general, a fin de construir sólidas conceptualizaciones con relación a las mismas, pues tal como lo dice Ardila (2014), "La implementación de nuevas tecnologías en Educación ha abierto un campo de posibilidades que requieren de la investigación y reflexión de los docentes” (p. 13).

Por otra parte, se concluye que el tema de las TIC se ha desarrollado de manera incipiente en programas de las universidades referenciadas, ya que de los 1014 TG en ellas realizados, tan solo 43 abordaron esta temática como objeto de estudio, lo que ni siquiera representa el $10 \%$ del total de las investigaciones. Sin embargo, una situación particular se registró en la Universidad Tecnológica de Pereira, en donde se encontró una gran producción con relación a las TIC, ya que 33 TG de los 369 en ella desarrollados corresponden a esta temática, lo que se debe en parte a la amplia trayectoria de este Centro de Formación en este campo del conocimiento.

Asimismo, se pudo evidenciar que en los TG analizados subyace una concepción instrumental de las TIC, situación que se puede develar a partir de la forma como se refieren a éstas en los textos analizados, ya que en ocasiones las TIC son asumidas como herramientas, en otros momentos como artefactos tecnológicos, así como recursos tecnológicos o medios para lograr un fin, siempre relacionando las TIC con elementos tangibles. 
Pareciera que dicha concepción instrumental de las TIC puede tener diferentes causas, una de ellas es el direccionamiento que el Gobierno Nacional ha dado a las políticas sobre TIC en el país, ya que por un lado se han planteado exigencias con relación a las TIC que los Centros de Formación Docente deben cumplir para obtener el Registro Calificado de sus programas, desconociendo la realidad de estos, que en ocasiones no cuentan con los recursos humanos ni físicos para tales fines; asimismo los esfuerzos del gobierno han estado enfocados mucho más a proveer dispositivos tecnológicos y acceso a Internet a los centros educativos, olvidando que estos dispositivos por sí solos no garantizan el aprendizaje.

Otra de las causas es el desconocimiento y el bajo dominio pedagógico que los docentes tienen de las TIC, pues aun cuando en la actualidad están más familiarizados con la tecnología y disfrutan utilizándola, no consiguen integrarla de manera eficaz al quehacer del aula, por lo que se hace necesario que los Centros de Formación Docente incluyan en sus planes de estudio el conocimiento en TIC que los maestros requieren, desde una verdadera comprensión y apropiación de ellas, a fin de que los docentes dominen no solo el carácter instrumental de las TIC mediante el uso de diferentes dispositivos tecnológicos, hasta la formación pedagógica que les permita reconocer la pertinencia de su uso en determinado momento, a fin de facilitar el proceso de enseñanza aprendizaje. Solo de esta forma se podrá superar la concepción instrumental que se ha construido con relación a las TIC en muchos de los actores educativos. 
Las TIC en la investigación de las Licenciaturas en Educación Infantil en Colombia

Molina Manosalva y Tobos Valderrama

\section{| Referencias}

Abadía, G., Quiceno, N., \& Rivera, K. (2013). Incidencia de la Enseñanza Mediada por TIC para Mejorar la Comprensión Lectora en los Niños de Grado Primero de la Institución Ciudad Boquía de Pereira. [Trabajo de grado, Universidad Tecnológica de Pereira]. http:// repositorio.utp.edu.co/dspace/bitstream/handle/11059/3695/37133A116. pdf? sequence=1\&isAllowed $=y$

Ardila, L. (2014). Tecno-Arte Actividades de Educación Artística Mediadas por la Tecnología. [Trabajo de grado, Universidad de la Sabana]. https://intellectum.unisabana.edu.co/handle/10818/15620?show=full

Avilez, K. (2018). Incorporación y Apropiación de Tecnologías Digitales en Colegios de Bogotá: La Oportunidad para Leer las Prácticas Pedagógicas. [Trabajo de grado, Pontificia Universidad Javeriana]. https://repository. javeriana.edu.co/handle/10554/40153

Bustos, E., Gómez, N., \& Galeano, A. (2019). El aula virtual como mediación para fortalecer el proceso de enseñanza y aprendizaje de la lengua extranjera inglés en los estudiantes del curso 204 de la IED Gerardo Paredes. [Trabajo de grado, Universidad Tecnológica de Pereira]. http://repository.pedagogica.edu.co/handle/20.500.12209/10278

Camacho, Y., \& Contreras, L. (2016). Comprensión y Apropiación de las Tecnologías de la Información y Comunicación - TIC en Docentes en Formación para la Infancia. Reflexión Pedagógica en Torno al Currículo de la Licenciatura en Educación Infantil en la Universidad Pedagógica Nacional. [Trabajo de grado, Universidad Pedagógica Nacional]. http://repository.pedagogica.edu.co/handle/20.500.12209/2405

Correa, M., \& Cuello, M. (2016). Infancia y socialización mediática: el papel de las tecnologías de la información y la comunicación (Tic) en la socialización de niños y niñas de 4 y 5 años. [Trabajo de grado, Universidad de Antioquia]. http://ayura.udea.edu.co:8080/jspui/ bitstream/123456789/2189/1/CA0213_\%20melisaCorrea_mairacuello_ infanciasicializacion.pdf 
Escorcia, L., \& Gómez, N. (2019). Aplicativo educativo digital para enseñanza del lenguaje en primera infancia. [Trabajo de grado, Pontificia Universidad Javeriana]. https://repository.javeriana.edu.co/ bitstream/handle/10554/43295/Entrega\%20tesis\%20u\%c4\%9bltima\%20 versi\%c3\%b3n\%20PDF.pdf? sequence $=4 \&$ is Allowed $=y$

Gadamer, H. (1993). Verdad y método. Fundamentos de una hermenéutica filosófica. Ediciones Sígueme.

Gil, J., \& Maldonado, H. (2009). Algunas implicaciones de integración de la ciencia y la tecnología en el diseño curricular del preescolar. [Trabajo de grado, Pontificia Universidad Javeriana]. https://repository. javeriana.edu.co/handle/10554/6659

Glasser, B., \& Strauss, A. (1967). The Discovery of Grounded Theory. Aldine Press.

Lamilla, A., Rodríguez, N., \& Salgado, M. (2015). Implicaciones de la Implementación de una Secuencia Didáctica Mediada por TIC en la Enseñanza y Aprendizaje de los Sistemas Geométricos en Grado Primero de Básica Primaria. [Trabajo de grado, Universidad Tecnológica de Pereira]. http://repositorio.utp.edu.co/dspace/handle/11059/5607

Lara, D. (2007). Análisis de la relación infancia y Tecnologías de la Información y la Comunicación, en el tiempo de ocio de niños y niñas, en las interacciones en la Biblioteca José Félix de Restrepo. [Trabajo de grado, Universidad de Antioquia]. http://ayura.udea.edu.co:8080/jspui/ bitstream/123456789/2592/1/CA0580_danielalara.pdf

Longoria, M. (2008). El uso de las Tics en la asesoría técnica de educación especial en el Estado de Chihuahua (México) como estrategia de mejora y optimización del servicio. [Tesis doctoral, Universidad de Salamanca] https://gredos.usal.es/bitstream/handle/10366/18454/DDOMI_Uso\%20 TICs\%20asesoria\%20tecnica\%20educacion $\% 20$ especial.pdf?sequence=1

Najar, O. (2016). Tecnologías de la información y la comunicación aplicadas a la educación. Praxis \& Saber, 7(14), 9-16. https://revistas.uptc.edu. co/index.php/praxis_saber/article/view/5215 
Parra, C. (2012). TIC, Conocimiento, Educación y Competencias Tecnológicas en la Formación de Maestros. Nomadas, 36, 144- 159. https://www. redalyc.org/pdf/1051/105124264010.pdf

Reyes, C. (2016). Propuesta Pedagógica para Promover la Educación en Tecnología en el Centro Aeiotú Nogal. [Trabajo de grado, Universidad Pedagógica Nacional]. http://repository.pedagogica.edu.co/ handle/20.500.12209/2492

Ricoeur, P. (1991). Del texto a la acción: Ensayos de hermenéutica II. Fondo de Cultura Económica.

Strauss A. \& Corbin J. (2002). Bases de la investigación cualitativa: Técnicas $y$ procedimientos para desarrollar la teoría fundada. Editorial Universidad de Antioquia. https://diversidadlocal.files.wordpress. com/2012/09/bases-investigacion-cualitativa.pdf

Schleiermacher, F. (2007). Acerca del concepto de hermenéutica con referencia a las indicaciones de F. A. Wolf y al manual de Ast. Philosophica, 31(1), 109-124

Tobos, M. (2016). Religación de la Práctica en el Proceso Formativo para los Programas de Formación Inicial de Profesionales de la Educación. [Tesis doctoral, Universidad del Atlántico].

Valencia, A. (2015). ¿Qué pasa en las interacciones cuando se vincula la tecnología en el diseño de la situación didáctica? Análisis de casos. [Trabajo de grado, Pontificia Universidad Javeriana]. https://repository. javeriana.edu.co/handle/10554/16836

Yepes, L. (2014). Percepciones de los futuros docentes sobre el uso de la tecnología y la robótica en el aula. [Trabajo de grado, Universidad de la Sabana]. https://intellectum.unisabana.edu.co/handle/10818/17247 